\title{
POSITIVE SOLUTIONS OF SINGULAR NONLINEAR STURM-LIOUVILLE BOUNDARY VALUE PROBLEMS
}

\author{
YAN SUN ${ }^{1}$, LISHAN LIU ${ }^{1}$ and YEOL JE CHO ${ }^{2}$
}

(Received 8 November, 2002; revised 29 August, 2003)

\begin{abstract}
By using fixed point index theory, we present the existence of positive solutions for a Sturm-Liouville singular boundary value problem with at least one positive solution. Our results significantly extend and improve many known results even for non-singular cases.
\end{abstract}

\section{Introduction}

In this paper, we show the existence of positive solutions for the following SturmLiouville singular boundary value problem (BVP):

$$
\left\{\begin{array}{l}
\frac{1}{p(t)}\left(p(t) u^{\prime}(t)\right)^{\prime}+\lambda g(t) F(t, u)=0, \quad 0<t<1, \\
\alpha u(0)-\lim _{t \rightarrow 0+} \beta p(t) u^{\prime}(t)=0, \\
\gamma u(1)+\lim _{t \rightarrow 1-} \delta p(t) u^{\prime}(t)=0,
\end{array}\right.
$$

where $\alpha, \beta, \gamma, \delta \geq 0, \beta \gamma+\alpha \gamma+\alpha \delta>0, \lambda>0, F:[0,1] \times[0,+\infty) \rightarrow[0,+\infty)$ is a continuous function and $p(t)$ and $g(t)$ may be singular at $t=0$ and/or $t=1$.

This problem arises in a variety of applications and the existence of positive solutions is very important (see $[1,7-9,12]$ for references along these lines). If $p(t)=1$, $\lambda g(t) F(t, x)=a(t) x^{p}$ for $0<p<1, \beta=\delta=0$ and $\alpha=\gamma=1$, BVP (1.1) becomes the well-known Emden-Fowler equation with a Dirichlet boundary value condition. In [12], using the method of lower and upper solutions, Zhang obtained

\footnotetext{
${ }^{1}$ Department of Mathematics, Qufu Normal University, Qufu, Shandong 273165, P. R. China; e-mail: ysun@163169.net, lls@qfnu.edu.cn.

${ }^{2}$ Department of Mathematics Education, The Research Institute of Natural Sciences, College of Education, Gyeongsang National University, Chinju 660-701, Korea; e-mail: yjcho@nongae.gsnu.ac.kr. (C) Australian Mathematical Society 2004, Serial-fee code 1446-1811/04
} 
a necessary and sufficient condition for the existence of positive solutions for the Emden-Fowler equation with a Dirichlet boundary value condition which is a special case of BVP (1.1). In [3] and [6], Dalmasso, and Ha and Lee supposed some monotone conditions on $F(t, x)$ to prove the existence of positive solutions for BVP (1.1) in a suitable subinterval for $\lambda$. In [9] and $[3,4,6,7,10]$, the authors proved their main results (when $p(t)=1$ and under suitable boundary value conditions) by using the norm-type cone expansion and compression theorem and under the assumption that $F(t, x)$ is either superlinear or is sublinear.

In this paper, without any monotone assumptions imposed on $F(t, x)$ with respect to $x$, we consider a more general BVP (1.1) with more general boundary conditions. We also allow $p(t)$ and $g(t)$ to have suitable singularities (such as $t=0$ and/or $t=1$ ). Thus the aim of this paper is to obtain at least one positive solution for BVP (1.1) in an explicit interval for any $\lambda$ and then we show how to apply our theorems to prove the existence of positive solutions to the Sturm-Liouville singular BVP. Our results extend and improve many known results in $[4,7-10,12]$.

By a positive solution of BVP (1.1), we mean a function $u \in C\left([0,1], R^{+}\right) \bigcap$ $C^{1}\left((0,1), R^{+}\right)$with $p(t) u^{\prime}(t) \in C^{1}\left((0,1), R^{+}\right)$satisfying BVP (1.1) with $u$ being nonnegative and not identically zero on $[0,1]$. If, for a particular $\lambda, B V P(1.1)$ has a positive solution $u$, the $\lambda$ is called an eigenvalue and $u$ a corresponding eigenfunction of BVP (1.1).

We adopt the following assumptions:

$\left(H_{1}\right) \quad p \in C^{1}((0,1),(0,+\infty)), 0<\int_{0}^{1} d t / p(t)<+\infty$;

$\left(H_{2}\right) \quad F(t, x) \in C([0,1] \times[0,+\infty),[0,+\infty)), g(t) \in C((0,1),[0,+\infty))$ and

$$
0<\int_{0}^{1} G(t, t) p(t) g(t) d t<+\infty,
$$

where $G(t, s)$ is Green's function for

$$
\left\{\begin{array}{l}
\frac{1}{p(t)}\left(p(t) u^{\prime}(t)\right)^{\prime}=0, \quad 0<t<1, \\
\alpha u(0)-\lim _{t \rightarrow 0+} \beta p(t) u^{\prime}(t)=0, \\
\gamma u(1)+\lim _{t \rightarrow 1-} \delta p(t) u^{\prime}(t)=0,
\end{array}\right.
$$

that is,

$$
G(t, s)= \begin{cases}\frac{1}{\rho}(\beta+\alpha B(0, s))(\delta+\gamma B(t, 1)), & 0 \leq s \leq t \leq 1, \\ \frac{1}{\rho}(\beta+\alpha B(0, t))(\delta+\gamma B(s, 1)), & 0 \leq t \leq s \leq 1,\end{cases}
$$

where $B(t, s)=\int_{t}^{s} d v / p(v)$ and $\rho=\alpha \delta+\alpha \gamma B(0,1)+\beta \gamma$ 
This paper is organised as follows. In Section 2, we present some properties of Green's functions (1.2) to be used in defining a positive operator. Also, we give some preliminaries. In Section 3, we give our main results and applications. We will give an appropriate Banach space and construct a cone applying the fixed point index theorem to show the existence of positive solutions for BVP (1.1) in an open interval of eigenvalues. In Section 4, we give our discussions on the conditions of the main theorems. We state some fixed point index theorems which will be needed in this paper. The following Lemmas 1.1 and 1.2 can be found in [5].

LEMMA 1.1. Let $K$ be a positive cone in real Banach space $E, \Omega$ be a bounded open set of $E, \theta \in \Omega$ and $T: K \cap \bar{\Omega} \rightarrow K$ be completely continuous (or more generally, condensing). Suppose that $T u \neq \mu u$ for any $u \in K \cap \partial \Omega$ and $\mu \geq 1$. Then $i(T, K \cap \Omega, K)=1$.

LEMMA 1.2. Let $K$ be a positive cone in real Banach space $E$ and $\Omega$ be a bounded open set of $E$. Let $T: K \cap \bar{\Omega} \rightarrow K$ be completely continuous and suppose that

(i) $\inf _{u \in K n a \Omega}\|T u\|>0$,

(ii) $T u \neq \mu u$ for any $u \in K \cap \partial \Omega$ and $0<\mu \leq 1$.

Then $i(T, K \cap \Omega, K)=0$.

\section{Some preliminaries}

For Green's function (1.2), it is easily to verify the following properties:

(I) $G(t, s) \leq G(s, s) \leq(\beta+\alpha B(0,1))(\delta+\gamma B(0,1)) / \rho<+\infty$, for any $t, s \in[0,1]$.

(II) If $[a, b] \subset(0,1)$, then $G(t, s) \geq(\beta+\alpha B(0, a))(\delta+\gamma B(b, 1)) / \rho>0$ for any $t, s \in[a, b]$.

(III) $G(t, s) \geq \omega G(s, s)$ for any $t \in[a, b] \subset(0,1)$ and $s \in[0,1]$, where

$$
\omega=\min \left\{\frac{\delta+\gamma B(b, 1)}{\delta+\gamma B(0,1)}, \frac{\beta+\alpha B(0, a)}{\beta+\alpha B(0,1)}\right\} .
$$

Note that it is easy to check that $0<\omega<1$.

REMARK 2.1. It follows from (I), $\left(H_{1}\right)$ and $\left(H_{2}\right)$ that there exist $0<a<b<1$ such that

$$
\begin{aligned}
0<\int_{a}^{b} p(t) d t & <+\infty, \quad 0<\min _{t \in[a, b]} \int_{a}^{b} G(t, s) p(s) g(s) d s<+\infty, \\
0 & <\max _{t \in[0,11} \int_{0}^{1} G(t, s) p(s) g(s) d s<+\infty .
\end{aligned}
$$


In the rest of this paper, $a$ and $b$ will be taken this way and we denote

$$
\begin{aligned}
l & =\min _{t \in[a, b]} \int_{a}^{b} G(t, s) p(s) g(s) d s \quad \text { and } \\
L & =\max _{t \in[0,1]} \int_{0}^{1} G(t, s) p(s) g(s) d s .
\end{aligned}
$$

Note that $0<l \leq L<+\infty$. Now, let

$$
\begin{gathered}
E=C[0,1], \quad C^{+}[0,1]=\{u \in C[0,1] \mid u \geq 0\}, \\
K=\left\{u \in C^{+}[0,1] \mid \min _{t \in[a, b]} u(t) \geq \omega\|u\|\right\},
\end{gathered}
$$

where $\omega$ is the constant defined by $(2.1)$ and $\|u\|=\sup _{t \in[0,1]}|u(t)|$. It is easy to see that $K$ is a cone in $C[0,1]$ and $K \subset C^{+}[0,1]$.

From the condition $\left(\mathrm{H}_{2}\right)$ and the property (I) of Green's function (1.2), we may define an operator $T: C^{+}[0,1] \rightarrow C^{+}[0,1]$ by

$$
(T u)(t)=\lambda \int_{0}^{1} G(t, s) p(s) g(s) F(s, u(s)) d s .
$$

It is well known that $u$ is a solution of BVP (1.1) if and only if $u \in K$ is a fixed point of the operator $T$.

LEMMA 2.1. Assume that the conditions $\left(H_{1}\right)-\left(H_{2}\right)$ hold. Then $T: C^{+}[0,1] \rightarrow$ $C^{+}[0,1]$ is a completely continuous operator.

PROOF. By the Lebesgue dominated convergence theorem, it is easy to prove that $T: C^{+}[0,1] \rightarrow C^{+}[0,1]$ is continuous and

$$
\begin{aligned}
(T u)^{\prime}(t)= & -\frac{\gamma}{\rho}\left(\lambda \int_{0}^{t}(\beta+\alpha B(0, s)) p(s) g(s) F(s, u(s)) d s\right) \\
& +\frac{\alpha}{\rho}\left(\lambda \int_{t}^{1}(\delta+\gamma B(s, 1)) p(s) g(s) F(s, u(s)) d s\right) .
\end{aligned}
$$

If $g(t) \in C[0,1]$ and $F(t, x) \in C([0,1] \times[0, \infty))$, then we can see that (2.2) implies that $T: C^{+}[0,1] \rightarrow C^{+}[0,1]$ is compact.

We now discuss a more general situation about $g(t) \in C(0,1)$ and $F(t, x) \in$ $C([0,1] \times[0, \infty))$. We define a function, for $n \geq 2$, by

$$
g_{n}(t)= \begin{cases}\inf \{g(t), g(1 / n)\}, & \text { if } 0<t \leq 1 / n, \\ g(t), & \text { if } 1 / n \leq t \leq(n-1) / n, \\ \inf \{g(t), g(1 / n)\}, & \text { if }(n-1) / n \leq t<1\end{cases}
$$


We define an operator $T_{n}: C^{+}[0,1] \rightarrow C^{+}[0,1]$ by

$$
\left(T_{n} u\right)(t)=\lambda \int_{0}^{1} G(t, s) p(s) g_{n}(s) F(s, u(s)) d s,
$$

where $n \geq 2, u \in C[0,1]$ and $t \in[0,1]$. Obviously, $T_{n}$ is compact on $C^{+}[0,1]$ for any $n \geq 2$. Denote $B_{R}=\left\{u \in C^{+}[0,1] \mid\|u\| \leq R\right\}$. Then $\left\{T_{n}\right\}$ converges uniformly to $T$ as $n \rightarrow+\infty$. In fact, for any $R>0, t \in[0,1]$ and $u \in B_{R}$, it follows from $\left(H_{2}\right)$ that

$$
\begin{aligned}
\left|\left(T_{n} u\right)(t)-(T u)(t)\right| \\
=\left|\int_{0}^{1} \lambda G(t, s) p(s)\left[g(s)-g_{n}(s)\right] F(s, u(s)) d s\right| \\
\leq \frac{\lambda}{\rho}(\delta+\gamma B(t, 1)) \int_{0}^{1 / n}(\beta+\alpha B(0, s)) p(s)\left|g(s)-g_{n}(s)\right| F(s, u(s)) d s \\
\quad+\frac{\lambda}{\rho}(\beta+\alpha B(0, t)) \int_{(n-1) / n}^{1}(\delta+\gamma B(s, 1)) p(s)\left|g(s)-g_{n}(s)\right| F(s, u(s)) d s \\
\leq C(R) \cdot \lambda\left(\int_{0}^{1 / n}(\beta+\alpha B(0, s)) p(s)\left|g(s)-g_{n}(s)\right| d s\right. \\
\left.\quad+\int_{(n-1) / n}^{1}(\delta+\gamma B(s, 1)) p(s)\left|g(s)-g_{n}(s)\right| d s\right) \rightarrow 0, \quad n \rightarrow+\infty,
\end{aligned}
$$

where

$$
C(R)=\frac{1}{\rho} \max \{\delta+\gamma B(0,1), \beta+\alpha B(0,1)\} \max _{(t, x) \in[0,1] \times[0, R]} F(t, x),
$$

and we have used $G(t, s) \leq G(s, s)$ for $t, s \in[0,1]$. Hence $\left\{T_{n}\right\}$ converges uniformly to $T$ as $n \rightarrow \infty$ and so $T$ is completely continuous also. This completes the proof.

LEMMA 2.2. $T\left(C^{+}[0,1]\right) \subset K$ and then $T(K) \subset K$.

ProOF. For all $u \in C^{+}[0,1], t \in[0,1]$, we have

$$
\begin{aligned}
(T u)(t) & =\lambda \int_{0}^{1} G(t, s) p(s) g(s) F(s, u(s)) d s \\
& \leq \lambda \int_{0}^{1} G(s, s) p(s) g(s) F(s, u(s)) d s .
\end{aligned}
$$

Thus it follows that

$$
\|T u\| \leq \lambda \int_{0}^{1} G(s, s) p(s) g(s) F(s, u(s)) d s .
$$


On the other hand, by (III), we have

$$
\begin{aligned}
\min _{t \in[a, b]}(T u)(t) & =\min _{t \in[a, b]} \lambda \int_{0}^{1} G(t, s) p(s) g(s) F(s, u(s)) d s \\
& \geq \lambda \omega \int_{0}^{1} G(s, s) p(s) g(s) F(s, u(s)) d s .
\end{aligned}
$$

This implies $\min _{t \in[a, b]}(T u)(t) \geq \omega\|T u\|$ and thus $T u \in K$. Therefore we have $T\left(C^{+}[0,1]\right) \subset K$. This completes the proof.

\section{The main results and examples}

We now give our main theorems and some examples.

THEOREM 3.1. Assume that the conditions $\left(H_{1}\right)$ and $\left(H_{2}\right)$ are satisfied. In addition, assume that

$\left(\mathrm{H}_{3}\right)$

$$
\begin{aligned}
& 0 \leq F^{0}=\lim \sup _{x \rightarrow 0+} \max _{t \in[0,1]}(F(t, x) / x)<L^{-1}, \\
& 0<l^{-1}<F_{\infty}=\liminf _{x \rightarrow+\infty} \min _{t \in[a, b]}(F(t, x) / x) \leq+\infty .
\end{aligned}
$$

Then $B V P(1.1)$ has at least one positive solution in $K$ for any

$$
\lambda \in\left(\frac{1}{l F_{\infty}}, \frac{1}{L F^{0}}\right),
$$

where $L$ and $l$ are defined as in Remark 2.1.

Proof. Let $\lambda$ satisfy (3.1) and $\varepsilon_{1}>0$ be a real number such that $F_{\infty}-\varepsilon_{1}>0$ and

$$
\frac{1}{\left(F_{\infty}-\varepsilon_{1}\right) l}<\lambda<\frac{1}{\left(F^{0}+\varepsilon_{1}\right) L} .
$$

Next, by the first part of $\left(H_{3}\right)$, there exists $r_{1}>0$ such that

$$
F(t, x) \leq\left(F^{0}+\varepsilon_{1}\right) x \leq\left(F^{0}+\varepsilon_{1}\right) r_{1}<\left(L^{-1}+\varepsilon_{1}\right) r_{1}
$$

for all $t \in[0,1]$ and $0<x \leq r_{1}$.

Let $\Omega_{1}=\left\{u \in C[0,1] \mid\|u\|<r_{1}\right\}$. It follows from (3.3) that, for all $u \in K \cap \partial \Omega_{1}$,

$$
\begin{aligned}
\|T u\| & =\max _{t \in[0,1]} \lambda \int_{0}^{1} G(t, s) p(s) g(s) F(s, u(s)) d s \\
& \leq\left(F^{0}+\varepsilon_{1}\right) r_{1} \lambda \max _{t \in[0,1]} \int_{0}^{1} G(t, s) p(s) g(s) d s . \\
& <r_{1}=\|u\|,
\end{aligned}
$$


which implies that $T u \neq \mu u$ for all $u \in K \cap \partial \Omega_{1}$ and $\mu \geq 1$. It follows from Lemma 1.1 that

$$
i\left(T, K \cap \Omega_{1}, K\right)=1 .
$$

On the other hand, by the second part of $\left(H_{3}\right)$, there exists $r_{2}>0$ and $r_{2}>r_{1}>0$ such that $F(t, x)>\left(F_{\infty}-\varepsilon_{1}\right) x$ for all $x \geq \omega r_{2}$ and $a \leq t \leq b$. Let $\Omega_{2}=\{u \in$ $\left.C[0,1] \mid\|u\|<r_{2}\right\}$. Then we have $\min _{t \in[a, b]} u(t) \geq \omega\|u\|=\omega r_{2}$ for all $u \in K \cap \partial \Omega_{2}$.

We now prove that $T u \neq \mu u$ for all $u \in K \cap \partial \Omega_{2}$ and $0<\mu \leq 1$. In fact, if not, there exist $u_{0} \in K \cap \partial \Omega_{2}$ and $0<\mu_{0} \leq 1$ such that $T u_{0}=\mu_{0} u_{0}$. Let $\tau=\min _{t \in[a, b]} u_{0}(t)$. Then, for any $a \leq t \leq b$, and $u_{0} \in K \cap \partial \Omega_{2}$, we have $\tau \geq \omega r_{2}$ and

$$
\begin{aligned}
u_{0}(t)=\mu_{0}^{-1}\left(T u_{0}\right)(t) & =\mu_{0}^{-1} \lambda \int_{0}^{1} G(t, s) p(s) g(s) F\left(s, u_{0}(s)\right) d s \\
& \geq \lambda \int_{a}^{b} G(t, s) p(s) g(s) F\left(s, u_{0}(s)\right) d s \\
& >\lambda\left(F_{\infty}-\varepsilon_{1}\right) \int_{a}^{b} G(t, s) p(s) g(s) u_{0}(s) d s \\
& \geq \lambda\left(F_{\infty}-\varepsilon_{1}\right) \tau \int_{a}^{b} G(t, s) p(s) g(s) d s \\
& \geq \lambda\left(F_{\infty}-\varepsilon_{1}\right) \tau \min _{t \in[a, b]} \int_{a}^{b} G(t, s) p(s) g(s) d s \\
& >\tau
\end{aligned}
$$

which yields a contradiction $\tau>\tau$. It can also be seen from the previous calculation that, for any $u \in K \cap \partial \Omega_{2}$ and $a \leq t \leq b,(T u)(t) \geq \omega r_{2}$ and so

$$
\inf _{u \in K \cap \partial \Omega_{2}}\|T u\| \geq \omega r_{2}>0
$$

It follows from Lemma 1.2 that

$$
i\left(T, K \cap \Omega_{2}, K\right)=0
$$

Since $0 \in \overline{\Omega_{1}} \subset \Omega_{2}$, it follows from (3.4) and (3.5) that

$$
\begin{aligned}
i\left(T, K \cap\left(\Omega_{2} \backslash \overline{\Omega_{1}}\right), K\right) & =i\left(T, K \cap \Omega_{2}, K\right)-i\left(T, K \cap \Omega_{1}, K\right) \\
& =0-1=-1 .
\end{aligned}
$$

It follows from [5, Theorem 2.3.2] that $T$ has a fixed point $u^{*}$ in $K \cap\left(\Omega_{2} \backslash \overline{\Omega_{1}}\right)$ and that $u^{*}$ is a positive solution of BVP (1.1). This completes the proof. 
EXAMPLE 3.1. Consider the following second-order differential equation boundary value problem:

$$
\left\{\begin{array}{l}
\frac{\left(\sqrt[6]{t} u^{\prime}(t)\right)^{\prime}}{\sqrt[6]{t}}+\frac{\lambda}{\sqrt[3]{t}}\left[(1+t) u^{3}+\frac{2008 u^{4}}{1+u}+90\left|\sin u^{3}\right|\right]^{1 / 3}=0, \quad 0<t<1 \\
u(0)=u(1)=0
\end{array}\right.
$$

It is obvious that $g(t)=1 / \sqrt[3]{t}$ is singular at $t=0$. It is difficult to solve the problem using the results in $[4,8,10]$ or their extensions.

We now study this problem using Theorem 3.1. Let $p(t)=\sqrt[6]{t}, g(t)=1 / \sqrt[3]{t}$ and

$$
F(t, x)=\left((1+t) x^{3}+2008 \frac{x^{4}}{1+x}+90\left|\sin x^{3}\right|\right)^{1 / 3}, \quad 0<t<1
$$

It is easy to see that the conditions $\left(H_{1}\right)$ and $\left(H_{2}\right)$ hold, where $\alpha=\gamma=1$ and $\beta=\delta=0$. The Green's function of (3.7) is (by simple calculation)

$$
G(t, s)= \begin{cases}\frac{6}{5} s^{5 / 6}\left(1-t^{5 / 6}\right), & \text { if } 0 \leq s \leq t \leq 1 \\ \frac{6}{5} t^{5 / 6}\left(1-s^{5 / 6}\right), & \text { if } 0 \leq t \leq s \leq 1\end{cases}
$$

By taking a subinterval $[a, b]=[1 / 4,3 / 4]$, then we have

$$
\begin{aligned}
\limsup \max _{x \rightarrow 0+} \frac{F(t, x)}{x} & =\sqrt[3]{92}, \\
\liminf _{x \rightarrow+\infty} \min _{t \in[1 / 4,3 / 4 \mid} \frac{F(t, x)}{x} & =\sqrt[3]{2009.25} .
\end{aligned}
$$

We now compute $L$ and $l$ in Theorem 3.1. Since

$$
\begin{aligned}
\Phi(t) & =\int_{0}^{1} G(t, s) p(s) g(s) d s \\
& =\int_{0}^{t} \frac{6}{5} s^{5 / 6}\left(1-t^{5 / 6}\right) \frac{1}{\sqrt[6]{s}} d s+\int_{t}^{1} \frac{6}{5} t^{5 / 6}\left(1-s^{5 / 6}\right) \frac{1}{\sqrt[6]{s}} d s \\
& =\frac{18}{25}\left(1-t^{5 / 6}\right) t^{5 / 3}+\frac{18}{25}\left(-1+t^{5 / 6}\right)^{2} t^{5 / 6} \\
& =\frac{18}{25}\left(1-t^{5 / 6}\right) t^{5 / 6}
\end{aligned}
$$

we have

$$
L=\max _{t \in|0.1|} \Phi(t)=\frac{18}{25}\left(1-\left(\frac{1}{\sqrt[5]{22}}\right)^{5 / 6}\right)\left(\frac{1}{\sqrt[5]{22}}\right)^{5 / 6}=\frac{9}{50}=0.18
$$


On the other hand, we have

$$
\begin{aligned}
\varphi(t)= & \int_{1 / 4}^{3 / 4} G(t, s) p(s) g(s) d s \\
= & \int_{1 / 4}^{t} \frac{6}{5} s^{5 / 6}\left(1-t^{5 / 6}\right) \frac{1}{\sqrt[6]{s}} d s+\int_{t}^{3 / 4} \frac{6}{5} t^{5 / 6}\left(1-s^{5 / 6}\right) \frac{1}{\sqrt[6]{s}} d s \\
= & \frac{9\left(-1+t^{5 / 6}\right)}{100 \cdot 2^{1 / 3}}-\frac{18}{25}\left(-1+t^{5 / 6}\right)-\frac{9}{200}\left(-8 \cdot 2^{1 / 3} \cdot 3^{5 / 6}+3 \cdot 6^{2 / 3}\right) t^{5 / 6} \\
& +\frac{18}{25}\left(-2+t^{5 / 6}\right) t^{5 / 3} \\
= & -\frac{9}{200}\left(2^{2 / 3}+\left(-2^{2 / 3}-8 \cdot 2^{1 / 3} \cdot 3^{5 / 6}+3 \cdot 6^{2 / 3}\right) t^{5 / 6}+16 t^{5 / 3}\right)
\end{aligned}
$$

It is easy to verify that

$$
l=\min _{t \in[1 / 4,3 / 4]} \varphi(t)=\varphi(3 / 4)=\frac{9}{400}(-2 \cdot \sqrt[3]{4}-9 \sqrt{3}+\sqrt[6]{243}+6 \sqrt[3]{36})
$$

and, by Theorem 3.1 and (3.8)-(3.11), BVP (3.7) has at least one positive solution in $K$ for any

$$
\frac{400}{9(-2 \cdot \sqrt[3]{4}-9 \sqrt{3}+\sqrt[6]{243}+6 \cdot \sqrt[3]{36}) \sqrt[3]{2009.25}}<\lambda<\frac{50}{9 \sqrt[3]{92}}
$$

By simple computation, we have

$$
\begin{aligned}
\frac{400}{9(-2 \sqrt[3]{4}-9 \sqrt{3}+\sqrt[6]{243}+6 \sqrt[3]{36}) \sqrt[3]{2009.25}} & \approx 0.993168 \\
\frac{50}{9 \sqrt[3]{92}} & \approx 1.23064
\end{aligned}
$$

Thus it follows that the approximate subinterval about $\lambda$ is $(0.99317,1.2306)$. In particular, we can see that BVP (3.7) has at least one positive solution when $\lambda=1$.

REMARK 3.1. From Theorem 3.1, we can see that $F(t, x)$ need not be superlinear or sublinear. So our conclusion extends and improves the corresponding results in $[2,4,7,10]$. In fact, Theorem 3.1 still holds if one of the following conditions holds:

(i) $F_{\infty}=\infty, F^{0}>0, \lambda \in\left(0,1 / L F^{0}\right)$,

(ii) $F_{\infty}=\infty, F^{0}=0, \lambda \in(0,+\infty)$,

(iii) $F_{\infty}>l^{-1}>0, F^{0}=0, \lambda \in\left(1 / l F_{\infty},+\infty\right)$. 
THEOREM 3.2. Suppose that the conditions $\left(H_{1}\right)$ and $\left(H_{2}\right)$ hold. In addition, assume that

$$
\begin{aligned}
& 0 \leq F^{\infty}=\limsup _{x \rightarrow+\infty} \max _{t \in[0,1]} \frac{F(t, x)}{x}<L^{-1}, \\
& 0<l^{-1}<F_{0}=\liminf _{x \rightarrow 0+} \min _{t \in[a, b]} \frac{F(t, x)}{x} \leq+\infty .
\end{aligned}
$$

Then BVP (1.1) has at least one positive solution for each

$$
\lambda \in\left(1 / l F_{0}, 1 / L F^{\infty}\right),
$$

where $L$ and $l$ are defined as in Remark 2.1.

ProOF. Let $\lambda$ satisfy (3.12) and $\varepsilon_{2}>0$ be a real number such that

$$
\frac{1}{\left(F_{0}-\varepsilon_{2}\right) l}<\lambda<\frac{1}{\left(F^{\infty}+\varepsilon_{2}\right) L} \text {. }
$$

Let $H(t, x)=\sup _{s \in[0, x]} F(t, s)$, then $F(t, x) \leq H(t, x)$ and $H$ is increasing for $x \in[0,+\infty)$.

For any $\varepsilon>0$, by the first part of $\left(H_{4}\right)$, there exists $r_{0}>0$ such that

$$
F(t, x) \leq\left(F^{\infty}+\varepsilon\right) x, \quad x \geq r_{0}, 0 \leq t \leq 1,
$$

and hence

$$
F(t, x) \leq M_{0}+\left(F^{\infty}+\varepsilon\right) x, \quad x \geq 0,0 \leq t \leq 1,
$$

where $M_{0}=\max _{(t, x) \in[0,1] \times\left[0, r_{0}\right]} F(t, x)$. Thus we have

$$
\lim \sup \max _{x \rightarrow+\infty} \frac{H(t, x)}{x} \leq F^{\infty} \text {. }
$$

On the other hand, since $F(t, x) \leq H(t, x)$, then we get

$$
\limsup _{x \rightarrow+\infty} \max _{t \in[0,1]} \frac{H(t, x)}{x}=F^{\infty} \text {. }
$$

Therefore there exists $r_{3}$ such that $r_{3}>r_{0}>0$ and $H(t, x) \leq\left(F^{\infty}+\varepsilon_{2}\right) x$ for all $x \geq r_{3}$ and $0 \leq t \leq 1$.

Now, let $\Omega_{3}=\left\{u \in C[0,1] \mid\|u\|<r_{3}\right\}$. For all $u \in K \cap \partial \Omega_{3}$, we then obtain

$$
\begin{aligned}
\|T u\| & =\max _{t \in[0,1]} \lambda \int_{0}^{1} G(t, s) p(s) g(s) F(s, u(s)) d s \\
& \leq \max _{t \in[0,1]} \lambda \int_{0}^{1} G(t, s) p(s) g(s) H(s, u(s)) d s \\
& \leq \max _{t \in[0.1]} \lambda \int_{0}^{1} G(t, s) p(s) g(s) H\left(s, r_{3}\right) d s \\
& \leq \lambda\left(F^{\infty}+\varepsilon_{2}\right) r_{3} \max _{t \in[0.1]} \int_{0}^{1} G(t, s) p(s) g(s) d s<r_{3}=\|u\|,
\end{aligned}
$$


which implies that $T u \neq \mu u$ for all $u \in K \cap \partial \Omega_{3}$ and $\mu \geq 1$. It follows from Lemma 1.1 that

$$
i\left(T, K \cap \Omega_{3}, K\right)=1 .
$$

By the second part of $\left(H_{4}\right)$, let $r_{4}>0$ and $r_{4}<r_{3}$ be such that $F(t, x) \geq\left(F_{0}-\varepsilon_{2}\right) x$ for all $0<x \leq r_{4}$ and $a \leq t \leq b$. Let $\Omega_{4}=\left\{x \in C[0,1] \mid\|u\|<r_{4}\right\}$. Then we have $\max _{t \in[a, b]} u(t) \leq\|u\|=r_{4}$, for all $u \in K \cap \partial \Omega_{4}$.

We now prove that $T u \neq \mu u$ for all $u \in K \cap \partial \Omega_{4}$ and $0<\mu \leq 1$. In fact, if not, there exist $u_{0} \in K \cap \partial \Omega_{4}$ and $0<\mu_{0} \leq 1$ such that $T u_{0}=\mu_{0} u_{0}$. Let $\tau=\min _{t \in[a, b]} u_{0}(t)$. Then, for any $a \leq t \leq b$ and $u_{0} \in K \cap \partial \Omega_{4}$, we have

$$
\begin{aligned}
u_{0}(t) & =\mu_{0}^{-1}\left(T u_{0}\right)(t)=\mu_{0}^{-1} \lambda \int_{0}^{1} G(t, s) p(s) g(s) F\left(s, u_{0}(s)\right) d s \\
& \geq \lambda \int_{a}^{b} G(t, s) p(s) g(s) F\left(s, u_{0}(s)\right) d s \\
& \geq \lambda\left(F_{0}-\varepsilon_{2}\right) \int_{a}^{b} G(t, s) p(s) g(s) u_{0}(s) d s \\
& \geq \lambda\left(F_{0}-\varepsilon_{2}\right) \tau \int_{a}^{b} G(t, s) p(s) g(s) d s \\
& \geq \lambda\left(F_{0}-\varepsilon_{2}\right) \tau \min _{t \in[a, b]} \int_{a}^{b} G(t, s) p(s) g(s) d s>\tau .
\end{aligned}
$$

This implies that $\tau>\tau$, which is a contradiction. It can also be seen from the previous calculation that, for any $u \in K \cap \partial \Omega_{4}$ and $a \leq t \leq b,(T u)(t) \geq\|u\|$. Thus $\inf _{u \in K \cap \partial \Omega_{A}}\|T u\| \geq\|u\|>0$. It follows from Lemma 1.2 that

$$
i\left(T, K \cap \Omega_{4}, K\right)=0 .
$$

Since $0 \in \overline{\Omega_{4}} \subset \Omega_{3}$, it follows from (3.14) and (3.15) that

$$
\begin{aligned}
i\left(T, K \cap\left(\Omega_{3} \backslash \overline{\Omega_{4}}\right), K\right) & =i\left(T, K \cap \Omega_{3}, K\right)-i\left(T, K \cap \Omega_{4}, K\right) \\
& =1-0=1 .
\end{aligned}
$$

Therefore, it follows from [5, Theorem 2.3.2] that $T$ has a fixed point $u^{*}$ in $K \cap\left(\Omega_{3} \backslash \overline{\Omega_{4}}\right)$ and so $u^{*}$ is a positive solution of BVP (1.1). This completes the proof.

EXAMPLE 3.2. Consider the following second-order differential equation BVP:

$$
\left\{\begin{array}{l}
\sqrt{t}(1-t)\left(\frac{1}{\sqrt{t}(1-t)} u^{\prime}(t)\right)^{\prime} \\
\quad+\lambda \frac{1-t}{\sqrt[3]{t}}(4 t u+81 \ln (1+u)+|\sin u|)=0, \quad 0<t<1, \\
u(0)=u(1)=0 .
\end{array}\right.
$$


It is obvious that $p(t)=1 / \sqrt{t}(1-t)$ and $g(t)=(1-t) / \sqrt[3]{t}$ are singular at $t=0$ and/or 1 . It seems difficult to solve the problem using the results obtained by $[4,8,10]$ or their extensions.

Now we study BVP (3.17) making use of Theorem 3.2 (where we choose $\alpha=\gamma=$ $1, \beta=\delta=0$ ).

Let $F(t, x)=4 t x+81 \ln (1+x)+|\sin x|, 0<t<1$. The Green's function of BVP (3.17) is (by simple calculation)

$$
G(t, s)= \begin{cases}\frac{1}{15} s^{3 / 2}(5-3 s)\left(2-5 t^{3 / 2}+3 t^{5 / 2}\right), & \text { if } 0 \leq s \leq t \leq 1, \\ \frac{1}{15} t^{3 / 2}(5-3 t)\left(2-5 s^{3 / 2}+3 s^{5 / 2}\right), & \text { if } 0 \leq t \leq s \leq 1 .\end{cases}
$$

Notice that $0 \leq G(s, s) \leq 1$. It is easy to see that conditions $\left(H_{1}\right)$ and $\left(H_{2}\right)$ hold.

By taking the subinterval $[a, b]=[1 / 4,3 / 4]$, then we have

$$
\begin{aligned}
\limsup _{x \rightarrow+\infty} \max _{t \in[0,1]} \frac{F(t, x)}{x}=4, \\
\liminf _{x \rightarrow 0+} \min _{t \in[1 / 4,3 / 4]} \frac{F(t, x)}{x}=83 .
\end{aligned}
$$

We now compute $L$ and $l$ in Theorem 3.2. Let

$$
\begin{aligned}
\xi(t)= & \int_{0}^{l} G(t, s) p(s) g(s) d s \\
= & \frac{1}{15} \int_{0}^{t} s^{2 / 3}(5-3 s)\left(2-5 t^{3 / 2}+3 t^{5 / 2}\right) d s \\
& +\frac{1}{15} \int_{t}^{1} t^{3 / 2}(5-3 t)\left(2-5 s^{3 / 2}+3 s^{5 / 2}\right) s^{-5 / 6} d s \\
= & \frac{1}{15}\left(2-5 t^{3 / 2}+3 t^{5 / 2}\right) \int_{0}^{t} s^{2 / 3}(5-3 s) d s \\
& +\frac{1}{15} t^{3 / 2}(5-3 t) \int_{t}^{1}\left(2-5 s^{3 / 2}+3 s^{5 / 2}\right) s^{-5 / 6} d s \\
= & \frac{1}{15}\left(2-5 t^{3 / 2}+3 t^{5 / 2}\right)\left(3 t^{5 / 3}-\frac{9}{8} t^{8 / 3}\right) \\
& +\frac{1}{15} t^{3 / 2}(5-3 t)\left(\frac{81}{8}-12 t^{1 / 6}+3 t^{5 / 3}-\frac{9}{8} t^{8 / 3}\right) \\
= & \frac{9}{40}\left(15 t^{3 / 2}-16 t^{5 / 3}-9 t^{5 / 2}+10 t^{8 / 3}\right) .
\end{aligned}
$$

Then it follows that

$$
L=\max _{t \in[0.11} \xi(t)=\xi\left(\frac{387420489}{1073741824}\right) \approx \frac{0.30586 \times 10^{24}}{0.48357 \times 10^{25}} \approx 0.06325 .
$$


On the other hand, we have

$$
\begin{aligned}
\Psi(t)= & \int_{1 / 4}^{3 / 4} G(t, s) p(s) g(s) d s \\
= & \frac{1}{15} \int_{1 / 4}^{t} s^{2 / 3}(5-3 s)\left(2-5 t^{3 / 2}+3 t^{5 / 2}\right) d s \\
& +\frac{1}{15} \int_{t}^{3 / 4} t^{3 / 2}(5-3 t)\left(2-5 s^{3 / 2}+3 s^{5 / 2}\right) s^{-5 / 6} d s \\
= & \frac{1}{15}\left(2-5 t^{3 / 2}+3 t^{5 / 2}\right) \int_{1 / 4}^{t} s^{2 / 3}(5-3 s) d s \\
& +\frac{1}{15} t^{3 / 2}(5-3 t) \int_{t}^{3 / 4}\left(2-5 s^{3 / 2}+3 s^{5 / 2}\right) s^{-5 / 6} d s \\
= & \frac{1}{2560(52+25 \sqrt{3})}\left\{( 5 - 3 t ) t ^ { 3 / 2 } \left(48073 \cdot 2^{3 / 2} \cdot 3^{1 / 6}\right.\right. \\
& +22012 \cdot 6^{2 / 3}-2048(52+25 \sqrt{3}) t^{1 / 6} \\
& \left.\left.+512(52+25 \sqrt{3}) t^{5 / 3}-192(52+25 \sqrt{3}) t^{8 / 3}\right)\right\} \\
& +\frac{1}{15}\left(2-5 t^{3 / 2}+3 t^{5 / 2}\right)\left(-\frac{87}{256 \cdot 2^{1 / 3}}-\frac{3}{128}\left(-128 t^{5 / 3}+48 t^{8 / 3}\right)\right) .
\end{aligned}
$$

It is easy to verify that

$$
\begin{aligned}
l= & \min _{t \in[1 / 4,3 / 4]} \Psi(t) \\
= & \Psi(3 / 4) \\
= & \left(3 3 \sqrt { 3 } \left(48073 \cdot 2^{2 / 3} \cdot 3^{1 / 6}+22012 \cdot 6^{2 / 3}-1024 \cdot 2^{2 / 3} \cdot 3^{1 / 6}(52+25 \sqrt{3})\right.\right. \\
& \left.\left.+69 \cdot 6^{2 / 3}(52+25 \sqrt{3})\right)\right) /(81920(52+25 \sqrt{3})) \\
& +\frac{1}{15}\left(2-\frac{33 \sqrt{3}}{32}\right)\left(-\frac{87}{256 \cdot 2^{1 / 3}}-\frac{3}{128}\left(\frac{27 \cdot 3^{2 / 3}}{2 \cdot 2^{1 / 3}}-24 \cdot 6^{2 / 3}\right)\right) \\
\approx & 0.0151846 .
\end{aligned}
$$

It follows from Theorem 3.2 and (3.18)-(3.20) that BVP (3.17) has at least one positive solution for any

$$
\frac{1}{83 \times 0.0151846}<\lambda<\frac{1}{4 \times 0.06325}
$$

where

$$
\frac{1}{83 \times 0.0151846} \approx 0.793448 \text { and } \frac{1}{4 \times 0.06325} \approx 3.95257
$$


The approximate subinterval about $\lambda$ is therefore $(0.7935,3.952)$. In particular, we can see that BVP (3.17) has at least one positive solution, when $\lambda=1$.

REMARK 3.2. From the proof of Theorem 3.2, we can see the conclusion of Theorem 3.2 still holds if one of the following conditions holds:

(i) $F^{\infty}<L^{-1}, F_{0}=\infty, \lambda \in\left(0,1 / L F^{\infty}\right)$,

(ii) $F^{\infty}=0, F_{0}=+\infty, \lambda \in(0,+\infty)$,

(iii) $F^{\infty}=0, F_{0}>l^{-1}>0, \lambda \in\left(1 / l F_{0},+\infty\right)$.

REMARK 3.3. It seems to be difficult to prove our results using the norm-type expansion and compression theorem used in [4] and [10]. From Examples 3.1 and 3.2, we can show not only the existence of positive solutions of BVP (1.1), but also provide the subinterval about $\lambda$, which was not done in previous papers (see $[4,10])$.

REMARK 3.4. Note that, if $F$ is superlinear, that is, $F^{0}=0$ and $F_{\infty}=+\infty$ or sublinear, that is, $F_{0}=+\infty$ and $F^{\infty}=0$ for any $\lambda \in(0,+\infty)$, BVP (1.1) has at least one positive solution. In particular, if $p(t)=1$ and $g(t) F(t, x)=a(t) f(x)$, the conclusions of Theorems 3.1 and 3.2 hold. Thus we generalise the main results of $\mathrm{Ma}$ [10]. Our results still hold for the non-singular cases as in [4] and [7].

\section{Discussions on the conditions of theorems}

We discuss the conditions given in our main results. Clearly, if $\alpha^{2}+\beta^{2} \neq 0$ or $\gamma^{2}+\delta^{2} \neq 0$, then $\rho=\alpha \delta+\alpha \gamma B(0,1)+\beta \gamma>0$. We suppose that $g_{i}(t)$, $i=0,1, \ldots, n$, are nonnegative continuous functions on $(0,1)$. Let

$$
H=\left\{g(t) F(t, x) \mid g \text { and } F \text { satisfy the condition }\left(H_{2}\right)\right\} .
$$

Then we can easily show that the following conclusions hold:

(i) If $g_{i}(t) \in H$, then $g_{i}(t) F(t, x) \in H, \quad i=1,2, \ldots, n$.

(ii) If $g_{i}(t) F_{i}(t, x) \in H$, then we have $\sum_{i=1}^{m} g_{i}(t) F_{i}(t, x) \in H, i=1,2, \ldots, n$, $\max _{1 \leq i \leq n} g_{i}(t) F_{i}(t, x) \in H, \min _{1 \leq i \leq n} g_{i}(t) F_{i}(t, x) \in H$.

By the above discussion, we can see that the conditions given in Theorems 3.1 and 3.2 are rather loose.

For example, consider the following equation:

$$
\left\{\begin{array}{l}
u^{\prime \prime}+\lambda \sum_{i, j=1}^{n}\left(g_{i}(t)+g_{j}(t)\right) u^{\alpha_{i}}=0 \\
u(0)=u^{\prime}(1)=0
\end{array}\right.
$$

We obtain the following result. 
COROLLARY 4.1. Suppose that

(i) $0<\alpha_{i}<1, g_{i}(t)$ and $g_{j}(t)(i, j=1,2, \ldots, n)$ are nonnegative and continuous (unbounded) on $(0,1)$;

(ii) $\int_{0}^{1} G(t, t)\left(g_{i}(t)+g_{j}(t)\right) d t<+\infty, i, j=1,2, \ldots, n$.

Then $B V P(4.1)$ has at least one positive solution for any $\lambda \in(0,+\infty)$.

Obviously BVP (4.1) is a special case of BVP (1.1) and so this corollary can be obtained by Theorem 3.2 directly.

\section{Acknowledgements}

The authors thank the referee for his/her valuable suggestions and comments. The first and second authors were supported by the NSFC (19871048) and NSFSP (02BS119) and the third author was supported by the Korea Research Foundation under Grant KRF-2001-005-D00002.

\section{References}

[1] R. P. Agarwal and D. O'Regan, "Positive solutions to superlinear singular boundary value problems", J. Comput. Appl. Math. 88 (1998) 129-147.

[2] J. G. Cheng and Z. J. Zhang, "On the existence of positive solutions for a class of singular boundary value problems", Nonlinear Anal. 44 (2001) 645-655.

[3] R. Dalmasso, "Positive solutions of singular boundary value problems", Nonlinear Anal. 27 (1996) 645-652.

[4] L. H. Erbe and H. Wang, "On the existence of positive solutions of ordinary differential equations", Proc. Amer. Math. Soc. 120 (1994) 743-748.

[5] D. Guo and V. Lakshmikantham, Nonlinear problems in abstract cones (Academic Press, San Diego, 1988).

[6] K. S. Ha and Y. H. Lee, "Existence of multiple positive solutions of singular boundary value problems", Nonlinear Anal. 28 (1997) 1429-1438.

[7] J. Henderson and H. Wang, "Positive solutions for nonlinear eigenvalue problems", J. Math. Anal. Appl. 208 (1997) 252-259.

[8] K. Q. Lan and J. Webb, "Positive solutions of semilinear differential equations with singularities", J. Differential Equations 148 (1998) 407-421.

[9] R. G. Li and L. S. Liu, "Positive solutions of boundary value problems for second-order singular nonlinear differential equations", Appl. Math. Mech. 22 (2001) 495-500.

[10] R. Y. Ma, "Positive solutions of singular second-order boundary value problems", Acta Math. Sinica (N.S.) 14 (1998) 691-698.

[11] D. O'Regan, "Singular second-order boundary-value problems", Nonlinear Anal. 15 (1990) 10971109.

[12] Y. Zhang, "Positive solutions of singular sublinear Emden-Fowler boundary value problems", $J$. Math. Anal. Appl. 185 (1994) 215-222. 